\title{
MBE-Grown Long-wavelength Interband Cascade Lasers on InAs Substrates
}

\author{
Lu Li ${ }^{a}$, Hao Ye ${ }^{a}$, Yuchao Jiang ${ }^{a}$, Rui Q. Yang ${ }^{a}$, Joel C. Keay ${ }^{b}$, Tetsuya D. Mishima ${ }^{b}$, \\ Michael B. Santos ${ }^{\mathrm{b}}$, and Matthew B. Johnson ${ }^{\mathrm{b}}$ \\ ${ }^{a}$ School of Electrical and Computer Engineering, University of Oklahoma, Norman, OK 73019 \\ ${ }^{b}$ Homer L. Dodge Department of Physics and Astronomy, University of Oklahoma, Norman, OK 73019
}

\begin{abstract}
An interband cascade (IC) laser structure with an emission wavelength designed to be near $11 \mu \mathrm{m}$ was grown by molecular beam epitaxy on an InAs substrate. Defects and surface smoothness, as well as the overall crystalline quality, were characterized by optical microscopy, atomic force microscopy and X-ray diffraction. The maximum operating temperatures of a broad-area IC laser device in $\mathrm{CW}$ and pulsed modes were $97 \mathrm{~K}$ and $130 \mathrm{~K}$, respectively, at an emission wavelength of $11 \mu \mathrm{m}$, which is the longest wavelength among interband lasers based on III-V semiconductor materials. The pulsed threshold current density at $80 \mathrm{~K}$ was measured to be $72 \mathrm{~A} / \mathrm{cm}^{2}$. The performance of this IC laser device may be limited by intersubband absorption loss in the active region and an unidentified carrier leakage channel.
\end{abstract}

Keywords: A3. Molecular beam epitaxy; B1. Antimonides; B2. III-V materials; B3. Infrared devices; B3. Laser diodes;

\section{Introduction}

With increasing emission wavelength, conventional interband lasers suffer from the limitation of the bandgaps of available semiconductor alloys, the difficulty of material growth, and the increase in free-carrier absorption loss and Auger recombination. IV-VI semiconductor lasers that can operate at low temperatures in the long-wavelength

Page 1 of 11 
infrared region [1], have a relatively high threshold current and low output power, partly because IV-VI growth and device processing are still immature compared to those for the III-V material systems. Interband cascade (IC) lasers [2] that contain type-II quantum-well $(\mathrm{QW})$ active regions, as a counterpart to the well-known intersubband quantum cascade (QC) lasers [3], have the same advantages of a cascade configuration and the ability to tailor their wavelength without being directly limited by the bandgaps of the constituent materials. Furthermore, the power consumption of IC lasers can be much lower than that of QC lasers, which will benefit some applications especially where energy cost is a concern.

IC lasers made of InAs/GaSb/AISb materials on GaSb substrates have demonstrated superior performance in the wavelength range from 3 to $6 \mu \mathrm{m}$ [4]. However, extending to longer wavelengths is difficult because the cladding regions, which consist of hundreds of InAs/AISb superlattice (SL) layers, have to be significantly thickened to accommodate the longer optical wave decay length. These thick SL layers increase the complexity of the molecular beam epitaxy (MBE) growth, as well as the thermal resistance of the device due to the much lower thermal conductivity of SL layers compared to bulk binary materials. For longer wavelengths, IC lasers with InAs plasmon cladding layers grown on InAs substrates would have significant benefits because of the lower refractive index and the higher thermal conductivity of the InAs plasmon cladding layers. These benefits have been demonstrated with room temperature operation beyond $6 \mu \mathrm{m}$ [5], as well as lasing up to $10.4 \mu \mathrm{m}$ [6]. In this paper, we report the MBE growth of an InAs-based IC laser structure with an emission wavelength at $11 \mu \mathrm{m}$, the longest lasing wavelength achieved for III-V interband semiconductor lasers. The maximum operating temperatures reached for a broad-area device were 97 and $130 \mathrm{~K}$ in CW and pulsed mode, respectively.

\section{Epitaxial growth and material characterizations}

The IC laser structure was grown on an epi-ready S-doped $\left(n \sim 2.5 \times 10^{18} \mathrm{~cm}^{-3}\right) \operatorname{InAs}$ substrate using an Intevac GEN II MBE system equipped with a valved As cracker and an unvalved Sb cracker. The growth temperature was monitored by an IRCON Modline 3 infrared pyrometer, which was calibrated using the GaSb surface reconstruction 
transition from (1X3) to (2X5) [7]. The growth rates for group III sources (In, Ga and Al) were calibrated by intensity oscillations of the reflection high-energy electron diffraction (RHEED) patterns prior to the growth of the IC laser structure. Moderate growth rates were adopted to grow Ga- and Al-containing alloys, compared to a low growth rate $(0.22$ $\mathrm{ML} / \mathrm{s}$ ) for InAs, which was found to be optimum in our previous growths of laser structures [8]. The cracker zones for both $\mathrm{As}$ and $\mathrm{Sb}$ were kept at $900^{\circ} \mathrm{C}$ to provide stable beams mostly composed of $\mathrm{As}_{2}$ and $\mathrm{Sb}_{2}$, respectively. While the flux ratios of $\mathrm{Sb}_{2} / \mathrm{Ga}$ and $\mathrm{Sb}_{2} / \mathrm{Al}$ were both maintained around 3, the $\mathrm{As}_{2} / \mathrm{In}$ flux ratio was kept near 8 to achieve a slight $A s_{2}$ overpressure as determined by the observation of an As-rich (2X4) surface under RHEED observation. During growth, the As-valve position was kept constant and only the shutter was used to control the As flux for the different layers. The whole structure was grown at $400^{\circ} \mathrm{C}$ as a compromise between the optimal growth windows for the thick InAs layers and the ultra-thin $\ln A s / A I S b / G a(\operatorname{In}) S b$ layers in the cascade regions.

After the oxide desorption process described in Ref. [8] was completed, the growth was initiated with a $2.3 \mu \mathrm{m}$ highly $n$-doped $\left(\mathrm{Si}, \sim 7 \times 10^{18} \mathrm{~cm}^{-3}\right) \ln A$ s layer as a bottom plasmon waveguide cladding layer. Then a $(\sim 1.9 \mu \mathrm{m})$ unintentionally doped InAs separate confinement layer (SCL) was grown, followed by the 20 cascade stages ( 46.2 $\mathrm{nm}$ per stage) for achieving an optical gain that is sufficient to overcome the likely increased optical loss expected for the longer wavelength range. Next, a top ( 2.5 $\mu \mathrm{m})$ unintentionally doped InAs SCL was grown and the growth ended with $35 \mathrm{~nm}$ of highly $n$-doped $\left(\mathrm{Si}, \sim 7 \times 10^{18} \mathrm{~cm}^{-3}\right)$ InAs as the top contact layer. Each cascade stage was composed of an $n$-type electron injector made of $\operatorname{lnAs} / \mathrm{AlSb}$ multiple $\mathrm{QWs}$, an active region (InAs/Ga $\mathrm{Ga}_{0.65} \mathrm{In}_{0.35} \mathrm{Sb} / \mathrm{InAs}, 38 / 28 / 33.5 \AA$, similar to "W"-shape $\mathrm{QWs}$ [9]) and a hole injector (GaSb/AISb QWs). Because InAs has the smallest lattice constant among all the alloys in the IC laser structure, strain balance for the whole structure to the InAs substrate was achieved by using AIAs interfaces in the electron/hole injection regions. Otherwise, there was no intentional shutter sequence at the interfaces between the ultra-thin alloy layers. Some of the InAs QWs in the electron injector were heavily $n$ doped to $\sim 2.3 \times 10^{18} \mathrm{~cm}^{-3}$ to rebalance the hole concentration [10]. The entire growth time exceeded 30 hours as the total thickness was close to $8 \mu \mathrm{m}$. 
After growth, the wafer was first assessed by differential interference contrast (DIC) microscopy for surface defects and texture. The surface defect density ranged from $7 \times 10^{3}$ to $2 \times 10^{4} \mathrm{~cm}^{-2}$ over the wafer. This density is comparable to the average etch pit density (EPD) of $\sim 1.5 \times 10^{4} \mathrm{~cm}^{-2}$ for the InAs substrate, as specified by the substrate supplier. Despite the long growth time for this thick structure, there was no appreciable increase in the defect density. Besides the limited defect density, the surface looks flat and featureless. The surface smoothness was quantified using an Asylum MFP-3D-BIO atomic force microscope (AFM). The AFM image (Fig. 1) shows uniform steps with atomic step edges, as expected from the InAs substrate miscut of $\sim 0.35^{\circ}$ from (100) toward (111)A. On the $2 \mu \mathrm{m} \times 2 \mu \mathrm{m}$ scan area the root-mean-square (RMS) roughness is only $0.1 \mathrm{~nm}$.

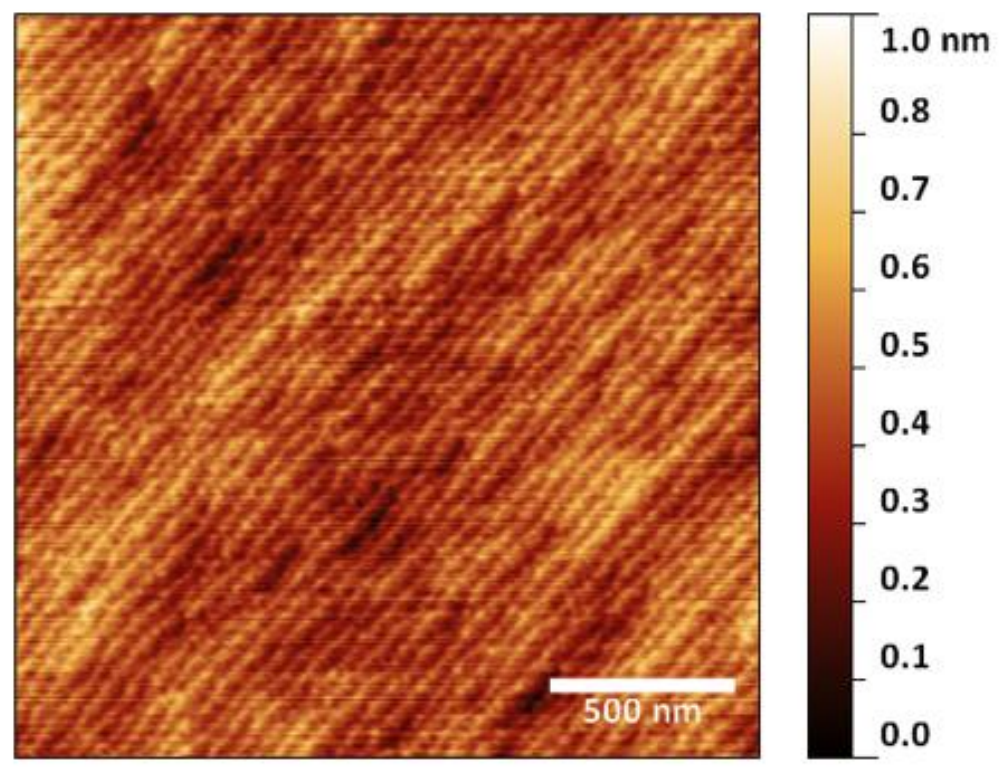

Fig. 1. A $2 \mu \mathrm{m} \times 2 \mu \mathrm{m}$ atomic force microscope image of the IC laser structure's surface.

The crystalline quality of the IC laser structure was characterized using a Philips MRD X-ray diffraction (XRD) system. Fig. 2 (top) shows experimental data from an XRD omega/2-theta scan around the InAs (004) reflection for the IC laser structure. Numerous sharp and narrow satellite peaks over $\pm 3^{\circ}$ around the InAs substrate peak were observed ( $>30$ orders at each side of the substrate peak with FWHM less than 30 
arcsec), indicating that the structure has an excellent crystalline quality. A simulated XRD curve (Fig. 2, bottom) based on the designed IC laser structure was calculated using a commercial XRD simulator (X'pert Epitaxy). The average thickness per stage in the cascade region of the IC laser structure $(47.5 \mathrm{~nm}$ as derived from the spacing of the satellite peaks) is only $3 \%$ more than design value. The experimental and simulated curves are nearly identical to each other, demonstrating that the MBE growth was well controlled over the long growth time.

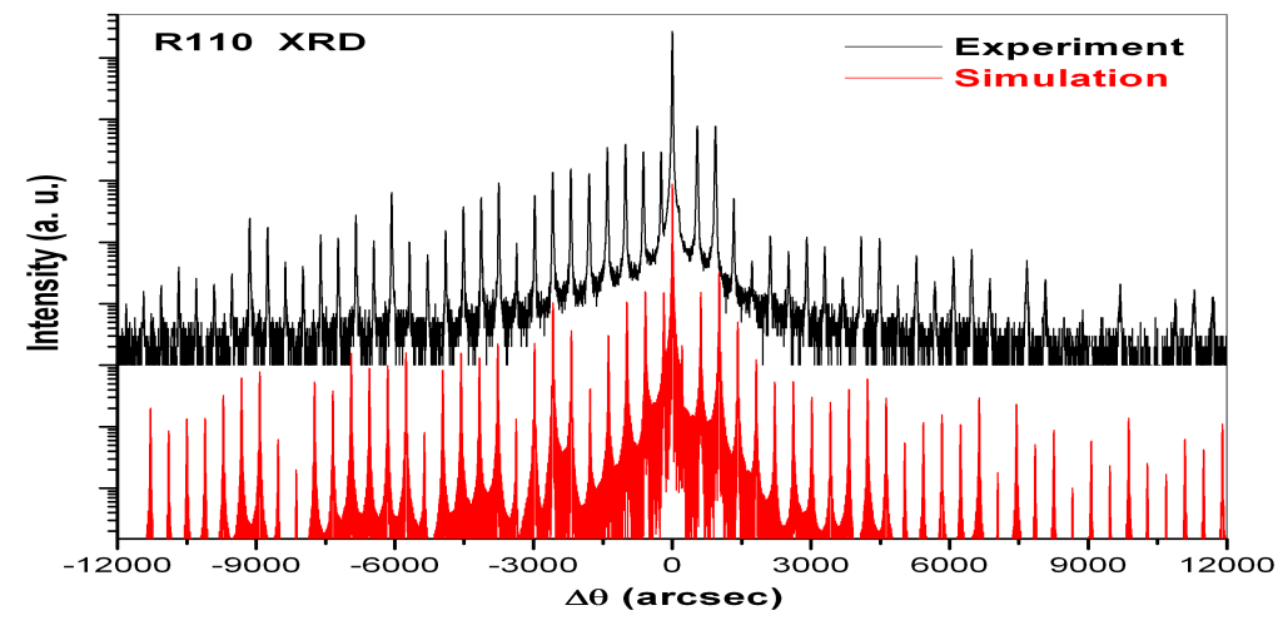

Fig. 2. (Top) Experimental XRD curve from an omega/2theta scan around the InAs (004) reflection for the IC laser structure. (Bottom) Corresponding simulated XRD curve calculated for the designed structure.

\section{Device results and discussion}

In order to reduce the influences of device processing and quickly evaluate the device performance, broad-area IC lasers were fabricated. The $150-\mu \mathrm{m}$-wide mesas were defined using UV contact lithography and wet-chemical etching. The etching proceeded to the bottom SCL to prevent lateral current spreading. A 220-nm-thick, 90- $\mu$ m-wide $\mathrm{SiO}_{2}$ insulating layer was deposited and lithographically defined (through lift-off) on the center of the mesa stripe, followed by the deposition and lithographic defining (through lift off) of a 30/300-nm-thick and $100-\mu \mathrm{m}$-wide Ti/Au layer that contacted the top InAs layer for current injection. After thinning the wafer down to $\sim 150 \mu \mathrm{m}$, the processing was 
finished by $\mathrm{Ti} / \mathrm{Au}$ metallization on the substrate side of the wafer. All the depositions were done using magnetron sputtering. A schematic of the processed broad-area device is shown in Fig. 3. The $\mathrm{SiO}_{2}$ insulating layer combined with the top metal layer, constitute the dielectric-metal hybrid top cladding layers used to confine optical wave [11]. Our waveguide simulation suggests that the confinement factor and free-carrier absorption loss in this 20-stage laser (without accounting for possible absorption loss due to intersubband transitions in the active region) are very similar to the previous 15 stage $9.1 \mu \mathrm{m} \mathrm{IC} \mathrm{lasers} \mathrm{reported} \mathrm{in} \mathrm{Ref.} \mathrm{[6].}$

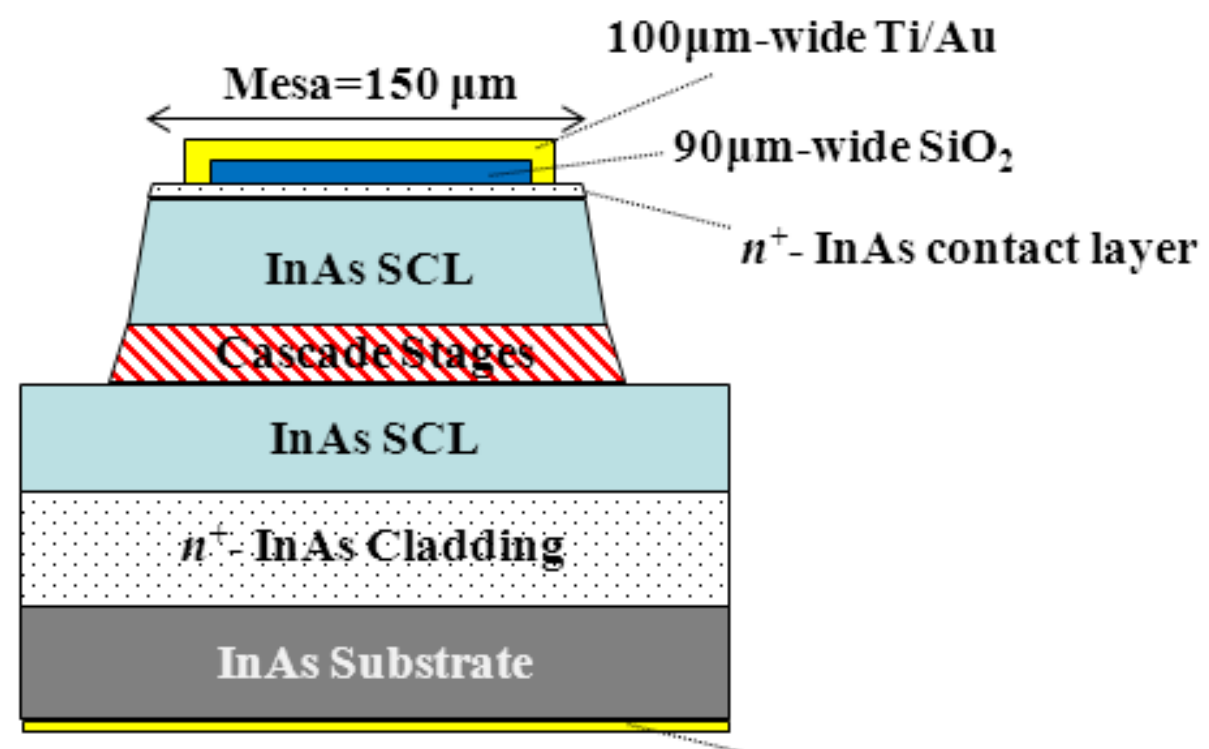

Backside Ti/Au contact

Fig. 3. Schematic of the processed broad-area IC laser.

The fabricated broad-area laser wafer was cleaved into bars with a cavity length ranging from 1 to $2 \mathrm{~mm}$, which were mounted epi-side up on a copper heat sink with indium solder and then wire bonded. The emission spectra were acquired by a Nicolet Fourier transform infrared (FTIR) spectrometer equipped with a liquid nitrogen cooled mercury cadmium telluride (MCT) detector. In CW mode, a 1.6-mm-long device lased near $10.8 \mu \mathrm{m}$ at $80 \mathrm{~K}$, and was operated at up to $97 \mathrm{~K}$ with an emission wavelength at $11.1 \mu \mathrm{m}$, as shown in the inset to Fig. 4. The threshold current density at $80 \mathrm{~K}$ in $\mathrm{CW}$ mode was $95 \mathrm{~A} / \mathrm{cm}^{2}$, much higher than the $\sim 10 \mathrm{~A} / \mathrm{cm}^{2}$ for IC lasers near $\sim 9 \mu \mathrm{m}$ 
operated under the same conditions [6]. The threshold voltage was $3.9 \mathrm{~V}$ at $80 \mathrm{~K}$, corresponding to a voltage efficiency of $61 \%$ that is lower than a typical value (e.g. $90 \%$ ) for InAs-based IC lasers.

Additionally, current-voltage characteristics exhibited some abnormal behavior - i.e., the slope that corresponds to the differential resistance had an abrupt drop well before the current reached the threshold, as shown in Fig. 4. Emission spectra were taken at several injection current levels around that point $(\sim 150 \mathrm{~mA})$ at $80 \mathrm{~K}$ to examine whether the device had lased or not. As shown in the inset to Fig. 4, electroluminescence peaks at currents below the threshold $(\sim 230 \mathrm{~mA})$ were broad $(>120 \mathrm{~nm})$ in contrast to the narrow lasing spectrum. This confirmed that the device did not lase before the current reached $230 \mathrm{~mA}$. Other IC lasers from the same wafer also exhibited an abrupt drop in differential resistance at various current densities before threshold. The devices, which had this pre-threshold drop in the differential resistance at smaller currents, had higher threshold currents, suggesting a correlation with defect related leakage and material non-uniformity. The abrupt drop in differential resistance might be caused by the activation of a carrier leakage channel, which increases the threshold current density and reduces the output power. The device at $80 \mathrm{~K}$ had an output power of 3.6 $\mathrm{mW} /$ facet at $600 \mathrm{~mA}$ (Fig. 4), which is much lower than our previous $9 \mu \mathrm{m}$ IC lasers [6].

Nevertheless, the threshold input power density $\left(370 \mathrm{~W} / \mathrm{cm}^{2}\right.$ in $\mathrm{CW}$ mode at $\left.80 \mathrm{~K}\right)$ was much lower than that of QC lasers at similar wavelengths [12, 13]. The threshold current density and voltage of $Q C$ lasers are typically around several hundreds of $A / \mathrm{cm}^{2}$ and 10 $V$, respectively. In pulsed mode ( $1 \mu \mathrm{s}$ at $5 \mathrm{kHz}$ ), our device lased at temperatures up to $130 \mathrm{~K}$ near $11.2 \mu \mathrm{m}$ (inset to Fig. 5), the longest wavelength achieved among III-V interband lasers. The light intensity did not increase rapidly immediately after reaching the threshold and the slope was initially small at the threshold as shown by the lightcurrent (L-I) characteristics in Fig. 5. Hence, it was difficult to accurately determine the threshold current by L-I curves. The threshold current was then obtained from the lasing spectra by varying current. At $80 \mathrm{~K}$, the threshold current density was $72 \mathrm{~A} / \mathrm{cm}^{2}$, which was about $24 \%$ lower than that in $\mathrm{cw}$ mode, suggesting substantial heating for broadarea lasers in $\mathrm{cw}$ operation even at $80 \mathrm{~K}$. 


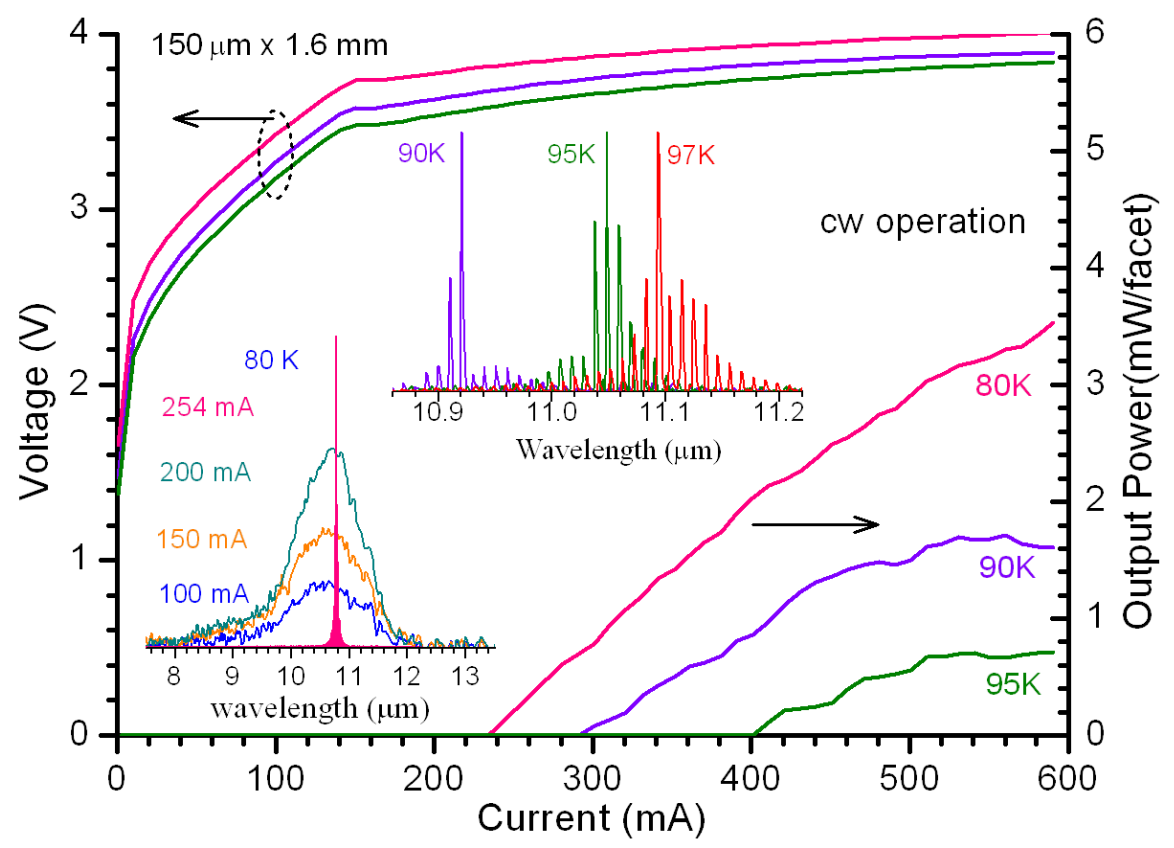

Fig. 4. Current-voltage-light characteristics for a $150-\mu \mathrm{m}$-wide device in cw operation. The insets show the $\mathrm{cw}$ lasing spectra at heat-sink temperatures of $80 \mathrm{~K}$ to $97 \mathrm{~K}$, and emission spectra at $80 \mathrm{~K}$ with several injection currents before threshold.

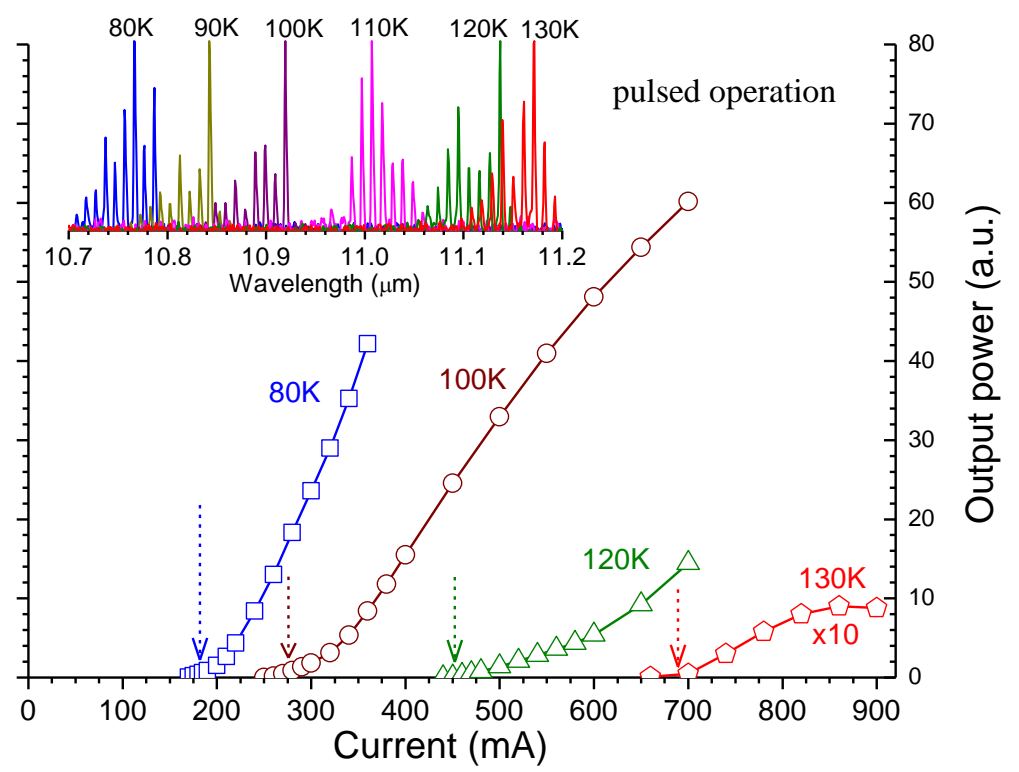

Fig. 5. Light-current curves for the broad-area IC laser in pulsed mode at temperatures of $80 \mathrm{~K}$ to $130 \mathrm{~K}$. The arrows point to threshold currents where spectra were taken. The inset shows the pulsed spectra at several temperatures. 
The $11 \mu \mathrm{m} \mathrm{IC} \mathrm{lasers} \mathrm{did} \mathrm{not} \mathrm{perform} \mathrm{as} \mathrm{well} \mathrm{as} \mathrm{our} \mathrm{previous} 9 \mu \mathrm{m} \mathrm{IC} \mathrm{lasers} \mathrm{in} \mathrm{terms} \mathrm{of}$ threshold current densities and output powers. The threshold current density can be expressed as: $J_{t h}=q\left(N_{t r}+\frac{\alpha_{i}+\alpha_{m}}{a \Gamma}\right) / \tau$, where $N_{t r}$ is the transparency carrier density, $\tau$ is the carrier lifetime, $\Gamma$ is the optical confinement factor, $a=d g / d N$ is the differential material gain, $\alpha_{m}$ is the mirror loss, and $\alpha_{i}$ is the internal loss which includes the waveguide loss (mainly free carrier absorption loss) and the absorption loss in the active region due possibly to intersubband transitions. The transparency carrier density depends on the band structure (see $[14,15]$ ) and is nearly insensitive to the lasing wavelength in type-II QWs at low temperatures (e.g. $80 \mathrm{~K}$ ), based on observed threshold current densities for type-II IC lasers over a wide spectral range. The typical transparency current density is small at low temperatures (e.g. $1-3 \mathrm{~A} / \mathrm{cm}^{2}$ at $80 \mathrm{~K}$ ). Hence, at $80 \mathrm{~K}$, there is a negligible difference between $N_{t r}$ for the $11 \mu \mathrm{m}$ and $9 \mu \mathrm{m}$ IC lasers, as their active regions are very similar. For the same reason and because the differential material gain, $d g / d N$, is proportional to the wavefunction overlap in the active region, the difference in $d g / d N$ between the $11 \mu \mathrm{m}$ and $9 \mu \mathrm{m}$ IC lasers would not be larger than $10 \%$. Considering the similar confinement factor and comparable mirror losses (because both their facets were uncoated), the much higher threshold current density in this $11 \mu \mathrm{m} \mathrm{IC} \mathrm{laser} \mathrm{might} \mathrm{be} \mathrm{caused} \mathrm{only} \mathrm{by} \mathrm{a} \mathrm{significantly} \mathrm{reduced} \mathrm{lifetime,} \tau$, and increased internal loss, $\alpha_{i}$. The reduced carrier lifetime could be related to more defects and an increased Auger recombination. However, a large increase in Auger recombination at $80 \mathrm{~K}$ is unlikely because the lasing wavelength was increased only from 9.1 to $10.8 \mu \mathrm{m}$, unless the threshold carrier concentration was greatly increased.

If the carrier leakage channel was not activated, an increase in threshold carrier concentration could only be caused by a considerable increase of internal optical loss based on the above discussion, which is consistent with the significant reduction of attainable output power. This is because the increased Auger recombination alone would not necessarily lower the output power, while the internal optical loss would do so. Hence, the lower performance of these long wavelength IC lasers could be primarily attributed to the combination of a possibly activated leakage channel and a large increase of internal optical loss beyond the common free-carrier absorption (the free- 
carrier absorption loss is similar to what was calculated in our $9 \mu \mathrm{m} \mathrm{IC} \mathrm{lasers).} \mathrm{Although}$ the cause of a high internal absorption loss is not clear at this stage, intersubband absorption loss in the active region is a possibility. Intersubband absorption loss occurs in QW structures [14], but in principle can be eliminated or alleviated by removing resonant intersubband transitions through quantum engineering. To fully understand and address this issue, further investigations are needed.

\section{Conclusions}

We have investigated the MBE growth of IC laser structures on InAs substrates, and demonstrated the longest lasing wavelength ever achieved by interband lasers based on III-V materials. Optical microscopy, AFM and XRD measurements showed that the material quality of the IC laser structure was quite good in terms of surface defects, smoothness and crystalline quality. A $150-\mu \mathrm{m} \times 1.6-\mathrm{mm}$ IC laser operated at $11 \mu \mathrm{m}$ at temperatures up to $97 \mathrm{~K}$ in $\mathrm{CW}$ mode and $130 \mathrm{~K}$ in pulsed mode. The threshold current density was noticeably higher and the output power lower than for our shorter wavelength IC lasers. This reduced performance was mainly attributed to a possible activated leakage channel and a significantly increased internal optical loss, not to the Auger recombination expected for increasing wavelength. At this stage, we do not fully understand why the threshold current density of this long wavelength IC laser was so much higher $(>7 x)$ with only a modest increase in lasing wavelength compared to our 9 $\mu \mathrm{m} I \mathrm{IC}$ lasers. Further investigations are required to achieving better understanding and improved device performance.

\section{Acknowledgements}

This work was supported by the National Science Foundation (ECCS-1002202 and IIP1346307), and by C-SPIN, the Oklahoma/Arkansas MRSEC (NSF DMR-0520550).

\section{References}

[1] E. Kapon, Semiconductor Lasers II: Materials and structures, in: Optics and Photonics, Academic Press, United States of America, 1998.

[2] R.Q. Yang, Superlattices Microstruct., 17 (1995) 77-83. 
[3] J. Faist, F. Capasso, D.L. Sivco, C. Sirtori, A.L. Hutchinson, A.Y. Cho, Science, 264 (1994) 553556.

[4] I. Vurgaftman, W.W. Bewley, C.L. Canedy, C.S. Kim, M. Kim, C.D. Merritt, J. Abell, J.R. Meyer, IEEE J. Sel. Topics Quantum Electron., 19 (2013) 1200210.

[5] M. Dallner, S. Höfling, M. Kamp, Electron. Lett., 49 (2013) 286-287.

[6] Z. Tian, L. Li, H. Ye, R.Q. Yang, T.D. Mishima, M.B. Santos, M.B. Johnson, Electron. Lett., 48 (2012) 113-114.

[7] A.S. Bracker, M.J. Yang, B.R. Bennett, J.C. Culbertson, W.J. Moore, J. Cryst. Growth, 220

(2000) 384-392.

[8] H. Ye, L. Li, R.T. Hinkey, R.Q. Yang, T.D. Mishima, J.C. Keay, M.B. Santos, M.B. Johnson, J. Vac.

Sci. Technol. B 31 (2013) 03C135.

[9] J.R. Meyer, C.A. Hoffman, F.J. Bartoli, L.R. Ram-Mohan, Appl. Phys. Lett., 67 (1995) 757-759.

[10] I. Vurgaftman, W.W. Bewley, C.L. Canedy, C.S. Kim, M. Kim, C.D. Merritt, J. Abell, J.R.

Lindle, J.R. Meyer, Nature communications, 2 (2011) 585.

[11] Z. Tian, Y. Jiang, L. Li, R.T. Hinkey, Z. Yin, R.Q. Yang, T.D. Mishima, M.B. Santos, M.B. Johnson, IEEE J. Quantum Electron., 48 (2012) 915.

[12] A. Tahraoui, A. Matlis, S. Slivken, J. Diaz, M. Razeghi, Appl. Phys. Lett., 78 (2001) 416-418.

[13] S. Slivken, Y. Bai, B. Gokden, S.R. Darvish, M. Razeghi, Proc. of SPIE, 7608 (2010) $76080 B$.

[14] Y.-M. Mu, R.Q. Yang, J. Appl. Phys., 84 (1998) 5357-5359.

[15] R.Q. Yang, Y.-M. Mu, SPIE, 3628 (1999) 104-112. 